\section{Epidermal growth factor-like domain multiple 7 (EGFL7): Expression and possible effect on biliary epithelium growth in cholangiocarcinoma}

\author{
Caterina L. Mammola, ${ }^{1}$ \\ Antonella Vetuschi, ${ }^{2}$ Luigi Pannarale, ${ }^{1}$ \\ Roberta Sferra, ${ }^{2}$ Romina Mancinelli ${ }^{1}$ \\ ${ }^{1}$ Department of Anatomical, \\ Histological, Forensic Medicine and \\ Orthopedics Sciences, Sapienza \\ University of Rome \\ ${ }^{2}$ Department of Biotechnological and \\ Applied Clinical Sciences, University of \\ L'Aquila, Italy
}

\begin{abstract}
Cholangiocarcinoma (CCA) is an aggressive biliary tract malignancy with limited treatment options and low survival rates. The intrahepatic subtype comprises two forms: mucin-iCCA and mixed-iCCA. Epidermal growth factor-like domain multiple (EGFL7) is overexpressed in less differentiated liver tumors. The aim of this study was to assess the presence of EGFL7 due to its possible role in the growth of CCA. Hematoxylin \& Eosin and periodic acidSchiff staining were used to evaluate the morphological aspects and glycogen deposition. Immunohistochemistry and immunofluorescence were performed to identify the presence of EGFL7 both in tumor sections ex vivo and in appropriate cell lines in culture. We found that EGFL7 is expressed in malignant cholangiocytes of mixed-iCCA and absent in mucin-iCCA. In conclusion the expression of EGFL7 might be useful in the classification of CCA subtypes.
\end{abstract}

\section{Introduction}

Liver cancer is one of the most common malignancies worldwide. Among them, hepatocellular carcinoma (HCC) and cholangiocarcinoma (CCA) are the two most common liver cancers. ${ }^{1,2}$ CCA represents a heterogeneous group of malignant neoplasms originating from damaged cholangiocytes, the epithelial cells that line the biliary tree. ${ }^{1,3-5}$ CCA can be classified into intrahepatic, perihilar or distal subtypes. ${ }^{6}$ Intrahepatic CCA (iCCA) is generally located proximally to the second-order bile ducts, perihilar CCA (pCCA) spreads between the second-order bile ducts and the attachment of the cystic duct into the common bile duct, and distal CCA (dCCA) grows in areas between the cystic duct and the ampulla of Vater. ${ }^{7-9}$ iCCA is characterized by two histological subtypes: originating from small or large intrahepatic bile ducts. ${ }^{10,11}$ The first type is a mixed iCCA showing an almost exclusively mass-forming growth pattern, ${ }^{12,13}$ whereas the second type is a mucinous iCCA and appears grossly as mass-forming, periductal infiltrating or intraductal growing. ${ }^{8}$ In contrast to multidisciplinary treatment strategies, overall 5-year survival rates for resectable intrahepatic CCA tumors remains between 30 and $35 \%$. For that reason, it is really crucial to investigate innovative targets to improve longterm survival for CCA. A number of angiogenic factors including vascular endothelial growth factor (VEGF) are overexpressed by CCA. ${ }^{14-16}$ EGFL7 is a secreted angiogenic factor which acts as a chemoattractant for endothelial cells. ${ }^{17}$ Recently, it has emerged as a factor regulating vascular development, exhibiting high level of expression during embryogenesis. ${ }^{18-20}$ Campagnolo et al. compared the expression of VEGF and EGFL7 in HCC and in surrounding cirrhotic tissue finding that VEGF levels were comparable, while EGFL7 showed a higher expression in moderately and less differentiated tumors. ${ }^{18}$ Considering that EGFL7 seems to play a key role in the undifferentiated phases of liver tumor formation and in its progression, our aim was to investigate the presence of EGFL7 in human CCA.

\section{Materials and Methods}

\section{Materials}

Reagents were mainly purchased from Sigma-Aldrich (St. Louis, MO, USA) and DBA ITALIA Srl (Milan, Italy). The rabbit polyclonal EGFL7 antibody was obtained from Proteintech Europe (Manchester, UK). The LSAB System-HRP used for IHC were purchased from Dako-Agilent (Santa Clara, CA, USA), while the specific secondary antibody Alexa Fluor 488 from Invitrogen (Carlsbad, CA, USA).

\section{Human samples}

Liver samples of fifteen human iCCA and peritumoral noncancerous tissue were obtained from patients submitted to surgical resection (aged 50 to 83 years), after informed consents, at the Department of Gastroenterology of the Academic General Hospital Umberto I, Sapienza University of Rome in accordance with the ethical standards and the Helsinki Declaration of 1975.
Correspondence: Romina Mancinelli, Department of Anatomical, Histological, Forensic Medicine and Orthopedics Sciences, Sapienza University of Rome, Via Alfonso Borelli 50, 00161 Rome, Italy.

Tel. +39.06.49918063; Fax: +39.06.49918062.

E- mail: romina.mancinelli@unitoma1.it

Key words: Liver; biliary epithelium; cholangiocytes; cholangiocarcinoma; EGFL7.

Contributions: CLM, worked on the study design and in the preliminary research reports about the studied CCAs, collected the several samples and helped in the experimental aspects; AV, RS, performed morphological and immunohistochemical analyses with critical text revision; LP, interpreted the results and worked on the discussion, formatted the manuscript as a brief report; RM, performed the immunofluorescence, interpreted the results, wrote the paper and elaborate the corresponding figures.

Funding: This project received financial support from "Progetti piccoli di Ateneo 2017" of Sapienza University of Rome.

Conflict of interest: The authors declare no conflict of interest.

Received for publication: 25 July 2018.

Accepted for publication: 14 November 2018

This work is licensed under a Creative Commons Attribution-NonCommercial 4.0 International License (CC BY-NC 4.0).

CCopyright C.L. Mammola et al., 2018

Licensee PAGEPress, Italy

European Journal of Histochemistry 2018; 62:2971 doi:10.4081/ejh.2018.2971

\section{Cell lines}

The study was performed in a human CCA cell line of extrahepatic origin: $\mathrm{Mz}$ ChA-1 and one of intrahepatic origin: $\mathrm{HuH}-$ 28 (gifts from Prof. G. Alpini - Texas A\&M University, TX, USA). ${ }^{21,22} \mathrm{We}$, also, used a human immortalized, nonmalignant, cholangiocyte cell line, H69. ${ }^{23}$ All the cell lines were maintained at $37^{\circ} \mathrm{C}$ in a $5 \% \mathrm{CO}_{2}$ incubator in the specific culture medium.

\section{Light microscopy: Morphologic study}

Specimens were immediately fixed in $10 \%$ buffered formalin and then dehydrated with alcohol, cleared in xylene and embedded in paraffin wax. Afterwards, the tissues were serially sectioned, obtaining $3 \mu \mathrm{m}$ thick sections that were stained with Hematoxylin \& Eosin (H\&E) and periodic acid-Schiff (PAS) staining system according to the standard protocols.

\section{Light microscopy: Immunohistoche- mistry}

For immunohistochemistry, endoge- 
nous peroxidase activity was blocked by incubation in hydrogen peroxide $(3 \%)$. Sections were then incubated overnight at $4^{\circ} \mathrm{C}$ with EGFL7 (1:100 Proteintech code 19291-1-AP). Samples were rinsed with PBS, incubated with secondary biotinylated antibody and then with Streptavidin-HRP (LSAB+ System-HRP). Diaminobenzidine (DAB) was used as chromogen, and sections were counterstained with Hematoxylin. For all immunoreactions, negative controls were also included. Immunohistochemical observations were taken by a light microscope Leica Microsystems DM 4500, with a camera Jenoptik ProgRes C10 Plus (Jena, Germany) and analyzed with an Image Analysis System (Delta Sistemi, Rome, Italy). We calculated the area occupied by EGFL7-positive cholangiocytes in the several iCCA samples, and expressed as a percentage with respect to the total duct mass. All counts were performed in at least 10 different areas at 20x magnification (from 3 different sections). Three researchers in a blinded fashion independently performed the evaluations.

\section{Light microscopy: Immunofluorescence}

Cholangiocytes from the several cell lines were seeded on coverslip in a 6 wellplate (500.000 cells per well). Immunofluorescence was performed by fixing cells in $4 \%$ paraformaldehyde, washing in PBS-T (PBS with $0.1 \%$ Tween 20) and incubating in $4 \%$ bovine serum albumin (BSA). Then, they were incubated with the previous primary antibody. After $1 \mathrm{~h}$, the cells were washed in PBS-T and placed in the specific Alexa Fluor 488 secondary antibody for 45 min. Finally, cholangiocytes were rinsed and mounted with a drop of DAPI. To demonstrate the specificity of the immunoreaction, negative controls were performed and the images were taken by the same microscope, the Leica DM 4500 with an $\mathrm{Hg}$ lamp powered by the separate ebq 100 supply unit and equipped with a turret for up to five fluorescence filter cubes, in the present study we used the filter cube DAPI/FITC/CY3.

\section{Statistical analysis}

Data are presented as arithmetic mean \pm standard deviation (SD). The differences between groups were analyzed by Student's $t$-test when two groups were analyzed. A Pvalue of $<0.05$ was considered statistically significant.
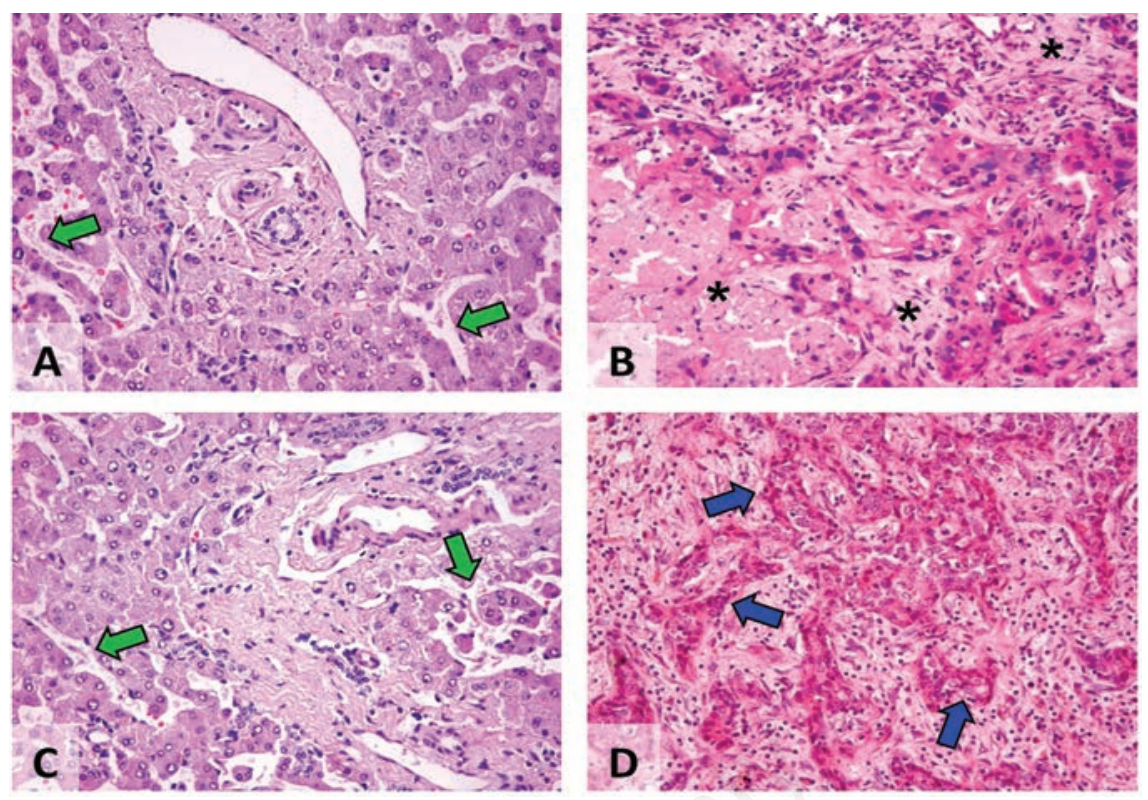

Figure 1. Representative images of H\&E staining of peritumoral tissue and CCA. A) The image shows a peritumoral area from mucin-iCCA; it presents a characteristic morphology except for the enlargements of the sinusoidal network (green arrows). B) Typical aspect of a mucin-iCCA with hepatic architecture destroyed and abundant stroma (asterisks); these tumor subtypes are formed by tubular, acinar or papillary structures, tumor cells are columnar with abundant clear or eosinophilic cytoplasm. C) Another image of perytumoral tissue, but from mixed-iCCA; we did not find any relevant differences among the several peritumoral tissues, where the parenchyma is characterized by similar enlargements of the sinusoids (green arrows). D) Representative area of a mixed-iCCA; bile ductular mixed-type iCCAs are constituted of areas with small tubular cord-like structures (blue arrows) with irregular lumina; the proportion of each area is largely variable. Original magnification: 20x.

A

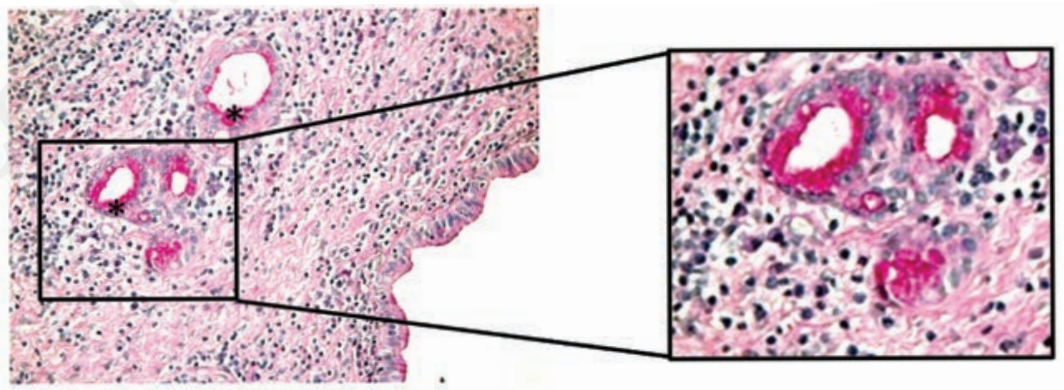

B

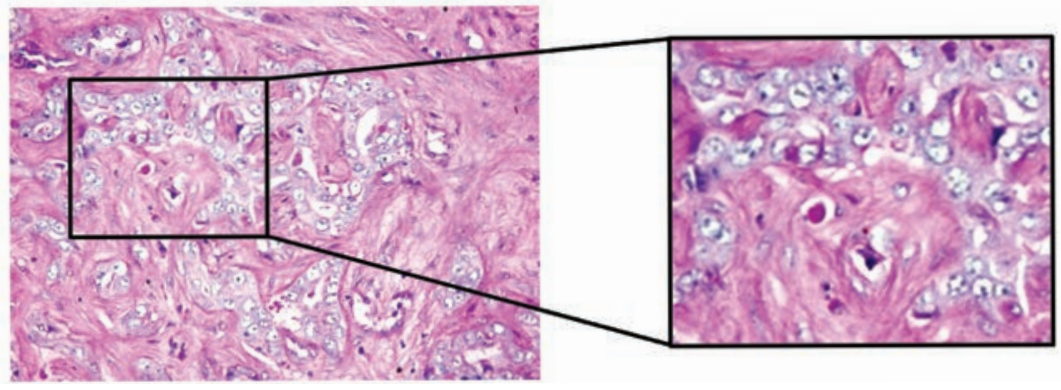

Figure 2. A) Typical image of mucin-type iCCA stained with PAS; these types of tumor are mostly composed of mucin-producing cells, as shown by the dark pink/purple color (asterisks); on the right, a higher magnification of a specific field to better show the mucin production. B) PAS stain of mixed-type iCCA; they display histological differences compared to the mucin-subtypes with very few focal mucin producing areas; on the right, a higher magnification of a specific area to indicate the decreased production of mucin. Original magnification: 20x. 


\section{Results}

\section{Tissue morphology}

H\&E staining of peritumoral tissues did not show significant differences between the several specimens from mucin-iCCAs (Figure 1A) and mixed-iCCAs (Figure 1C). They display parenchyma with scattered lipid droplets and the typical enlargements of the sinusoidal network (Figure 1A-C). In CCA samples, the morphology was totally changed with absence of the distinctive hepatic architecture, liver mass showing tubular adenocarcinoma in portal tissue, areas occupied by stromal components were quite predominating over those with epithelium. The mucin-iCCA subtypes contain tubular, acinar or papillary structures, tumor cells are columnar with abundant clear cytoplasm (Figure 1B). On the other hand, mixed-iCCA subtypes were characterized by areas of small tubular cord-like structures and irregular lumina variable in dimension, containing areas of hepatocytic differentiation (Figure 1D). Afterwards, we used PAS staining to highlight molecules with a high percentage of carbohydrate content such as mucin. Through PAS we differ- entiated six mucin-iCCA, PAS positive (Figure 2A) and nine samples as mixediCCA, PAS negative (Figure 2B). PAS positivity was widespread in the epithelial neoplastic regions of only mucin-iCCAs mostly composed of mucin-producing cells (largest biliary ducts) (Figure 2A). While, PAS negative samples showed histological diversity in mixed-type iCCAs mainly formed by mucin-negative cells (smallest biliary ducts) (Figure 2B).

\section{Expression of EGFL7 in the different CCA subtypes}

EGFL7 protein expression was very low in mucin-iCCA samples (Figure 3A), while mixed-iCCAs were characterized by a significant and specific increase of the angiogenic factor in cholangiocytes (Figure 3B). EGFL7 levels in mixed-iCCAs were increased 4-fold (43.2 \pm 9.4$)$, when compared with their levels in mucin-iCCAs $(10.2 \pm 4.8)$ with statistically significant differences $(\mathrm{P}=0.002)$. These data were confirmed in cell lines, where the CCA line arising from intrahepatic bile ducts (HuH28) showed the expression of EGFL7, whereas the other line of extrahepatic origin (Mz-ChA-1) as well as the non-malignant cholangiocyte line (H69) were lacking the angiogenic factor (Supplementary Figure 1).

\section{Discussion}

A number of angiogenic factors including VEGF and endothelin have been showed to play a key role in the growth of CCA. ${ }^{16,24}$ In particular, the overexpression of VEGF has been correlated to the 'angiogenic switch' of the malignant phenotype in human CCA. ${ }^{14,24}$ By contrast, endothelin-1 has an inhibitory effect on tumor growth with increased apoptosis of cholangiocytes. ${ }^{16,25,26}$ EGFL7, an attractive angiogenic factor, that may sustain hepatic tumor differentiation in parallel with VEGF. ${ }^{18,20,27}$ In the present study, we differentiated our CCAs in mixed-iCCA and mucin-iCCA through PAS staining. In fact, according to the World Health Organization (WHO) classification, ${ }^{28} \mathrm{CCAs}$ include a pure mucin-secreting and a mixed form. ${ }^{3}$ The mucin-iCCAs derive from the mucinsecreting epithelium lining large ducts formed by differentiated and mature cells; while, the mixed form arises from nonmucin-secreting cells lining small bile ducts or canals of Hering formed by immature cholangiocytes. ${ }^{28-30}$ Subsequently, we investigated the presence of EGFL7 in the different subtypes of CCAs finding a really inter-
Negative control

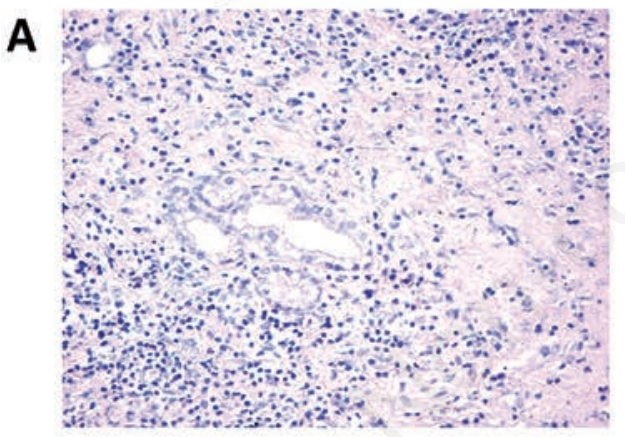

B

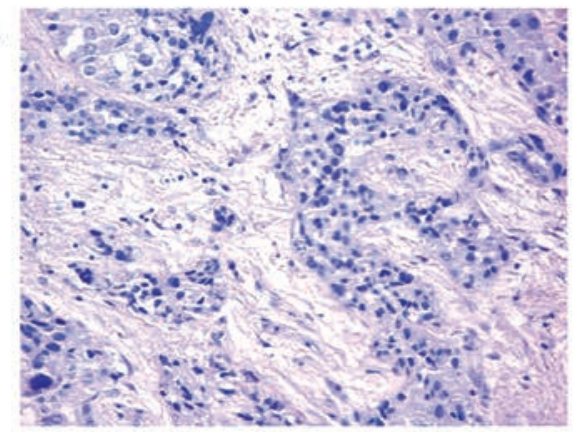

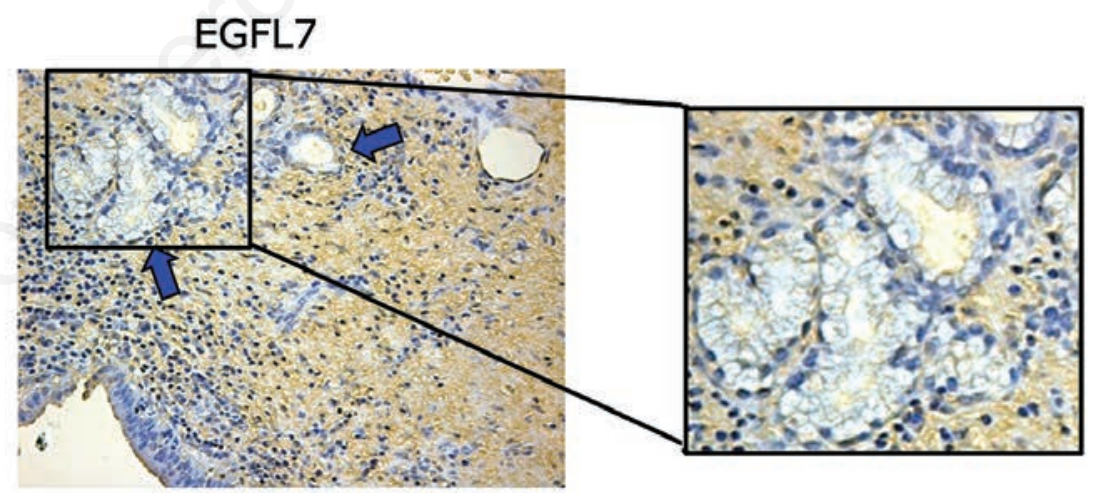

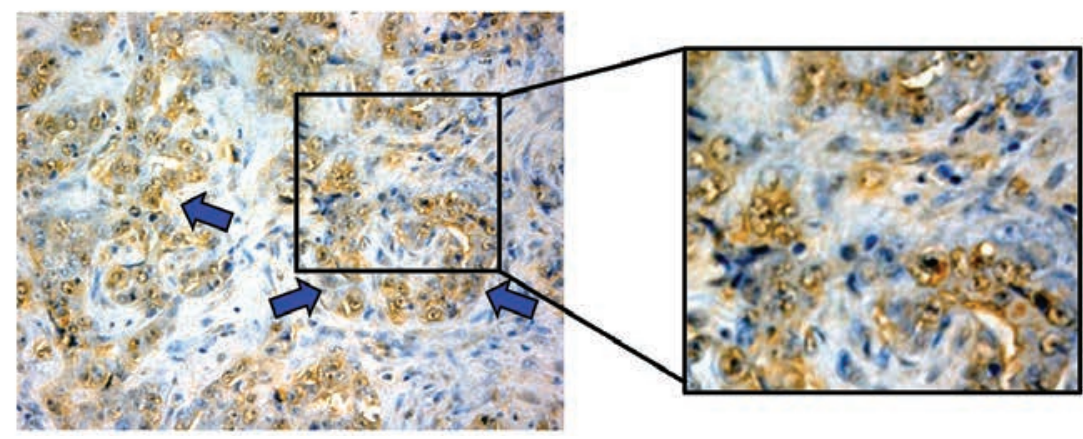

Figure 3. A) Immunohistochemistry for EGFL7 in mucin-type iCCAs; cholangiocytes are almost completely negative for the expression of EGFL7 in all the representative picture (blue arrows); on the left, representative picture of a negative control; on the right, a higher magnification of an area to show the absence of immunoreactivity on biliary epithelium. B) Immunohistochemistry for EGFL7 in mixed-type iCCAs; cholangiocytes are significantly positive for the presence of EGFL7 in all the representative pictures (blue arrows); on the left, representative picture of a negative control; on the right, a higher magnification of an area to display the specific and enhanced immunoreactivity on biliary epithelium. Original magnification: 20x. 
esting distinction, since the immunopositivity is very high and specific in cholangiocytes of mixed-iCCAs. In contrast, we found an almost complete absence of EGFL7 expression in mucin-iCCAs. This histological subtyping should be taken into serious consideration because it underlines different cell of origin, etiology, risk factors, molecular profile, clinical outcome and response to treatment. In fact, our data showed that CCAs arising from small and less differentiated cholangiocytes express EGFL7, whereas CCAs arising from large and well differentiated cells do not express EGFL7. Thus, since CCA is highly heterogeneous not only in initiation and location but also in progression, EGFL 7 expression may be really useful in the classification of the several subtypes of CCA considering its early presence in the growth of the tumor.

\section{References}

1. Munshi MK, Priester S, Gaudio E, Yang F, Alpini G, Mancinelli R, et al. Regulation of biliary proliferation by neuroendocrine factors: implications for the pathogenesis of cholestatic liver diseases. Am J Pathol 2011;178:472-84.

2. Yazici C, Niemeyer DJ, Iannitti DA, Russo MW. Hepatocellular carcinoma and cholangiocarcinoma: an update. Expert Rev Gastroenterol Hepatol 2014;8:63-82.

3. Cardinale V, Renzi A, Carpino G, Torrice A, Bragazzi MC, Giuliante F, et al. Profiles of cancer stem cell subpopulations in cholangiocarcinomas. Am J Pathol 2015;185:1724-39.

4. Rizvi S, Gores GJ. Pathogenesis, diagnosis, and management of cholangiocarcinoma. Gastroenterology 2013;145:1215-29.

5. Stratta P, Canavese C, Gurioli L, Porcu M, Todros T, Mattone GC, et al. Ratio between aldosterone and atrial natriuretic peptide in pregnancy. Kidney Int 1989; 36:908-14.

6. Oliveira IS, Kilcoyne A, Everett JM, Mino-Kenudson M, Harisinghani MG, Ganesan K. Cholangiocarcinoma: classification, diagnosis, staging, imaging features, and management. Abdom Radiol (NY) 2017;42:1637-49.

7. Kennedy L, Hargrove L, Demieville J, Francis N, Seils R, Villamaria S, et al. Recent Advances in understanding cholangiocarcinoma. F1000 Res 2017; 6:1818.

8. Cardinale V, Bragazzi MC, Carpino G, Torrice A, Fraveto A, Gentile R, et al. Cholangiocarcinoma: increasing burden of classifications. Hepatobiliary Surg Nutr 2013;2:272-80.

9. Banales JM, Cardinale V, Carpino G,
Marzioni M, Andersen JB, Invernizzi P, et al. Expert consensus document: Cholangiocarcinoma: current knowledge and future perspectives consensus statement from the European Network for the Study of Cholangiocarcinoma (ENSCCA). Nat Rev Gastroenterol Hepatol 2016;13:261-80.

10. Nakanuma Y, Sato Y, Harada K, Sasaki M, $\mathrm{Xu}$ J, Ikeda H. Pathological classification of intrahepatic cholangiocarcinoma based on a new concept. World J Hepatol 2010; 2:419-27.

11. Aishima S, Oda Y. Pathogenesis and classification of intrahepatic cholangiocarcinoma: different characters of perihilar large duct type versus peripheral small duct type. J Hepatobiliary Pancreat Sci 2015;22:94-100.

12. Nakanuma Y, Xu J, Harada K, Sato Y, Sasaki M, Ikeda $\mathrm{H}$, et al. Pathological spectrum of intrahepatic cholangiocarcinoma arising in non-biliary chronic advanced liver diseases. Pathol Int 2011;61:298305.

13. Liau JY, Tsai JH, Yuan RH, Chang CN, Lee HJ, Jeng YM. Morphological subclassification of intrahepatic cholangiocarcinoma: etiological, clinicopathological, and molecular features. Mod Pathol 2014; 27:1163-73.

14. Benckert C, Jonas S, Cramer T, Von Marschall Z, Schafer G, Peters M, et al. Transforming growth factor beta 1 stimulates vascular endothelial growth factor gene transcription in human cholangiocellular carcinoma cells. Cancer Res 2003; 63:1083-92.

15. Gaudio E, Barbaro B, Alvaro D, Glaser S, Francis H, Ueno Y, et al. Vascular endothelial growth factor stimulates rat cholangiocyte proliferation via an autocrine mechanism. Gastroenterology 2006;130: 1270-82.

16. Fava G, Demorrow S, Gaudio E, Franchitto A, Onori P, Carpino G, et al. Endothelin inhibits cholangiocarcinoma growth by a decrease in the vascular endothelial growth factor expression Liver Int 2009;29:1031-42.

17. Nichol D, Stuhlmann H. EGFL7: a unique angiogenic signaling factor in vascular development and disease. Blood 2012; 119:1345-52.

18. Campagnolo L, Telesca C, Massimiani M, Stuhlmann H, Angelico M, Lenci I, et al. Different expression of VEGF and EGFL7 in human hepatocellular carcinoma. Dig Liver Dis 2016;48:76-80.

19. Wu F, Yang LY, Li YF, Ou DP, Chen DP, Fan C. Novel role for epidermal growth factor-like domain 7 in metastasis of human hepatocellular carcinoma. Hepatology 2009;50:1839-50.

20. Fan C, Yang LY, Wu F, Tao YM, Liu LS,
Zhang JF, et al. The expression of Egfl7 in human normal tissues and epithelial tumors. Int J Biol Markers 2013;28:71-83.

21. Meng F, DeMorrow S, Venter J, Frampton G, Han Y, Francis H, et al. Overexpression of membrane metalloendopeptidase inhibits substance $\mathrm{P}$ stimulation of cholangiocarcinoma growth. Am J Physiol Gastrointest Liver Physiol 2014;306: G759-68.

22. Fava G, Marucci L, Glaser S, Francis H, De Morrow S, Benedetti A, et al. gammaAminobutyric acid inhibits cholangiocarcinoma growth by cyclic AMP-dependent regulation of the protein kinase $\mathrm{A} /$ extracellular signal-regulated kinase 1/2 pathway. Cancer Res 2005;65:11437-46.

23. Francis H, DeMorrow S, Venter J, Onori P, White M, Gaudio E, et al. Inhibition of histidine decarboxylase ablates the autocrine tumorigenic effects of histamine in human cholangiocarcinoma. Gut 2012; 61:753-64.

24. Mancino A, Mancino MG, Glaser SS, Alpini G, Bolognese A, Izzo L, et al. Estrogens stimulate the proliferation of human cholangiocarcinoma by inducing the expression and secretion of vascular endothelial growth factor. Dig Liver Dis 2009;41:156-63.

25. Laplante P, Raymond MA, Gagnon G, Vigneault N, Sasseville AM, Langelier Y, et al. Novel fibrogenic pathways are activated in response to endothelial apoptosis: implications in the pathophysiology of systemic sclerosis. J Immunol 2005; 174 : 5740-9.

26. Han Y, Onori P, Meng F, DeMorrow S, Venter J, Francis H, et al. Prolonged exposure of cholestatic rats to complete dark inhibits biliary hyperplasia and liver fibrosis. Am J Physiol Gastrointest Liver Physiol 2014;307:G894-904.

27. Braet F, Wisse E. Structural and functional aspects of liver sinusoidal endothelial cell fenestrae: a review. Comp Hepatol 2002;1:1.

28. Komuta M, Govaere O, Vandecaveye V, Akiba J, Van Steenbergen W, Verslype C, et al. Histological diversity in cholangiocellular carcinoma reflects the different cholangiocyte phenotypes. Hepatology 2012;55:1876-88.

29. Brunt E, Aishima S, Clavien PA, Fowler K, Goodman Z, Gores G, et al. cHCCCCA: Consensus terminology for primary liver carcinomas with both hepatocytic and cholangiocytic differentation. Hepatology 2018;68:113-26.

30. Mancinelli R, Franchitto A, Glaser S, Meng F, Onori P, Demorrow S, et al. GABA induces the differentiation of small into large cholangiocytes by activation of $\mathrm{Ca}(2+)$ /CaMK I-dependent adenylyl cyclase 8. Hepatology 2013;58:251-63. 